\title{
Long-Term Symptoms among COVID-19 Survivors in Prospective Cohort Study, Brazil
}

\author{
Lívia P. Bonifácio, Viviane N.F. Csizmar, Francisco Barbosa-Júnior, Ana P.S. Pereira, \\ Marcel Koenigkam-Santos, Danilo T. Wada, Gilberto G. Gaspar, Felipe S. Carvalho, \\ Valdes R. Bollela, Rodrigo C. Santana, João P. Souza, Fernando Bellissimo-Rodrigues
}

We conducted a prospective cohort study in a population with diverse ethnic backgrounds from Brazil to assess clinically meaningful symptoms after surviving coronavirus disease. For most of the 175 patients in the study, clinically meaningful symptoms, including fatigue, dyspnea, cough, headache, and muscle weakness, persisted for $\geq 120$ days after disease onset.

丁 nderstanding is growing that coronavirus disease (COVID-19) can evolve and continue to cause prolonged symptoms, characterizing the post-COVID-19 condition (1-3). Potential implications go beyond effects on individual patients and might represent an additional burden on healthcare services and social security, which are both already affected by the pandemic. Therefore, learning more about the long-term repercussions of the disease among different populations is essential. This study aimed to describe the occurrence of long-term physical, psychological, and social consequences among patients who survived COVID-19 and received follow-up care at a post-COVID-19 outpatient clinic at a university hospital in Brazil.

\section{The Study}

This prospective cohort study (RECOVIDA) was performed among patients attending a post-

Author affiliations: Ribeirão Preto Medical School Social Medicine Department, University of São Paulo, São Paulo, Brazil

(L.P. Bonifácio, V.N.F. Csizmar, F. Barbosa-Júnior, A.P.S. Pereira, J.P. Souza, F. Bellissimo-Rodrigues); Ribeirão Preto Medical

School Department of Medical Images, Hematology and Clinical Oncology, University of São Paulo, Ribeirão Preto, Brazil (M. Koenigkam-Santos, D.T. Wada); Ribeirão Preto Medical School Infectious and Tropical Diseases Division of Internal Medicine Department, University of São Paulo, Ribeirão Preto (G.G. Gaspar, F.S. Carvalho, V.R. Bollela, R.C. Santana)
COVID-19 outpatient clinic at Ribeirão Preto Medical School University Hospital, Ribeirão Preto, Brazil (4). The institutional review board approved the research protocol.

All adults with PCR-confirmed COVID-19 with symptom onset during February 1-December 31, 2020, who attended follow-up appointments at the study clinic were eligible. Most participants (85.7\%) had been discharged after being hospitalized for COVID-19. The remaining participants $(14.3 \%)$ were mostly healthcare workers from the study facility. No participants had been previously vaccinated against COVID-19. Patients were classified into 3 groups according to the World Health Organization (WHO) severity classification of COVID-19: mild/moderate, severe, and critical (5) (Appendix Table 1, https:/ / wwwnc.cdc.gov/EID/ article/28/3/21-2020-App1.pdf).

This study was exploratory, and sample size was established through convenience. We aimed to include all patients who attended the clinic during the study period and agreed to participate.

Participants were recruited just before the scheduled medical consultation. After the informed consent form was signed, we performed a structured interview and a brief physical examination. We obtained secondary data from patients' electronic health records. Laboratory and imaging tests were performed at the attending physician's clinical discretion. We collected study data by using the Research Electronic Data Capture platform (6).

We collected information on economic and demographic social profile, medical history, date of symptom onset, hospitalization data, laboratory and imaging test results, persistent symptoms, and quality of life. We assessed quality of life by using the WHO Quality of Life questionnaire (7-9) (Appendix). The date of symptom onset was used as the reference for follow-up. 
We performed statistical procedures by using Minitab 19.2 (https://www.minitab.com) and Stata version 9 (https://www.stata.com). We used odds ratios, 95\% CIs, and Fisher exact tests to verify the association between the persistence of symptoms and the severity of disease.

During the study period, 297 patients had a follow-up medical consultation scheduled at the outpatient clinic. We included 175 patients in this study (Table 1; Figure). In this sample, $20 \%$ of participants had illness that was considered mild/moderate, $45.7 \%$ were severe, and $34.3 \%$ were critical.

After COVID-19, 80\% of the patients experienced persistent symptoms; the 5 most prevalent were fatigue, dyspnea, cough, headache, and loss of overall muscle strength. Compared with the mild/moderate group, patients from the critical group more fre- quently experienced headaches, change in skin sensitivity, hypogeusia, hyposmia, and loss of muscle strength (Table 2, https://wwwnc.cdc.gov/EID/ article/28/3/21-2020-T2.htm).

Regarding quality of life after COVID-19, physical health was more severely affected than the other 3 domains evaluated by the WHO Quality of Life questionnaire (psychological, social relationships, and environmental). Moreover, the comparative evaluation before and after COVID-19 showed a decrease from $81.1 \%$ to $68.4 \%$ in the percentage of patients who believed that their quality of life was good or very good and an increase from $2.3 \%$ to $6.4 \%$ of those who believed that their quality of life was poor or very poor. Despite these changes, more than half of patients $(56.7 \%)$ were satisfied with their current health status at the time of evaluation (Appendix).

Table 1. Baseline clinical and demographic characteristics among 175 patients surviving the acute phase of COVID-19, Ribeirão Preto, Brazil $^{*}$

\begin{tabular}{|c|c|c|c|c|}
\hline \multirow[b]{2}{*}{ Characteristic } & \multicolumn{4}{|c|}{ COVID-19 severity } \\
\hline & $\begin{array}{c}\text { Mild/moderate, } \mathrm{n}=35 \\
(20 \%)\end{array}$ & $\begin{array}{c}\text { Severe, } n=80 \\
(45.7 \%)\end{array}$ & $\begin{array}{c}\text { Critical, } \mathrm{n}=60 \\
(34.3 \%)\end{array}$ & Total, $\mathrm{n}=175$ \\
\hline \multicolumn{5}{|l|}{ Sex } \\
\hline M & $7(20)$ & $36(45)$ & $42(70)$ & $85(48.6)$ \\
\hline $\mathrm{F}$ & $28(80)$ & $44(55)$ & $18(30)$ & $90(51.4)$ \\
\hline Mean age, y (SD) & $44.9( \pm 10.3)$ & $57.1( \pm 15.3)$ & $54.2( \pm 13.2)$ & $53.7( \pm 14.4)$ \\
\hline \multicolumn{5}{|l|}{ Ethnic background $\dagger$} \\
\hline White (Caucasian or Latin) & $19(54.3)$ & $36(45)$ & $25(41.7)$ & $80(45.7)$ \\
\hline Afro-American (Brown) & $10(28.6)$ & $34(42.5)$ & $26(43.3)$ & $70(40)$ \\
\hline Afro-American (Black) & $6(17.1)$ & $8(10)$ & $6(10)$ & $20(11.4)$ \\
\hline Asiatic & 0 & $1(1.3)$ & $2(3.3)$ & $3(1.7)$ \\
\hline Brazilian Indigenous & 0 & $1(1.3)$ & $1(1.7)$ & $2(1.1)$ \\
\hline Mean years of schooling (SD) & $13.4( \pm 5.7)$ & $8.1(+5.5)$ & $8.3(+5.4)$ & $9.2(+5.9)$ \\
\hline Mean income/person, USD (SD) $\ddagger$ & $407.33( \pm 313.60)$ & $273.01( \pm 295,85)$ & $229.33( \pm 210.40)$ & $285.57( \pm 279.56)$ \\
\hline Median & 364.01 & $200.21 \ddagger$ & $182.01 \ddagger$ & $216.77 \ddagger$ \\
\hline \multicolumn{5}{|l|}{ Currently works as a health professional } \\
\hline Yes & $23(65.7)$ & $8(10)$ & $2(3.3)$ & $33(18.9)$ \\
\hline No & $12(34.3)$ & $72(90)$ & $58(96.7)$ & $142(81.1)$ \\
\hline Mean BMI (SD)§ & $31.8( \pm 7.5)$ & $32.1( \pm 7.3) \S$ & $31.1( \pm 7.5)$ & $31.7( \pm 7.3) \S$ \\
\hline $\mathrm{BMI} \geq 30 \S$ & $17(48.6)$ & $44(56.4) \S$ & $23(38.3)$ & $84(48.6) \S$ \\
\hline \multicolumn{5}{|l|}{ Underlying conditions } \\
\hline None & $16(45.7)$ & $16(20)$ & $10(16.7)$ & $42(24.0)$ \\
\hline Hypertension & $9(25.7)$ & $35(43.8)$ & $21(35)$ & $65(37.1)$ \\
\hline Diabetes & $1(2.9)$ & $26(32.5)$ & $22(36.7)$ & $49(28.0)$ \\
\hline Dyslipidemia & $2(5.7)$ & $12(15)$ & $12(20)$ & $26(14.8)$ \\
\hline Heart problems (other than hypertension) & $1(2.9)$ & $10(12.5)$ & $8(13.3)$ & $19(10.9)$ \\
\hline Rhinitis or sinusitis & $3(8.6)$ & $7(8.8)$ & $7(11.7)$ & $17(9.7)$ \\
\hline Cancer & $1(2.9)$ & $9(11.3)$ & $1(1.7)$ & $11(6.3)$ \\
\hline Thyroid problems & 0 & $4(5)$ & $6(10)$ & $10(5.7)$ \\
\hline Depression or anxiety & $1(2.9)$ & $6(7.5)$ & $3(5)$ & $10(5.7)$ \\
\hline \multicolumn{5}{|l|}{ Smoking } \\
\hline Current & $0(0)$ & $2(2.5)$ & 0 & $2(1.1)$ \\
\hline Previous & $2(5.71)$ & $18(22.5)$ & $19(31.7)$ & $39(22.3)$ \\
\hline \multicolumn{5}{|l|}{ Hospitalization } \\
\hline Yes & $10(28.6)$ & $80(100)$ & $60(100)$ & $150(85.7)$ \\
\hline No & $25(71.4)$ & 0 & 0 & $25(14.3)$ \\
\hline Mean duration of hospitalization, $d(S D)$ & $5( \pm 4)$ & $9.9( \pm 5.2)$ & $24.1( \pm 11.1)$ & $15.3( \pm 10.9)$ \\
\hline Median & 4 & $\overline{9}$ & 20.5 & 12 \\
\hline
\end{tabular}




\section{Conclusions}

We describe the long-term repercussions of COVID-19 among a sample of patients in Brazil from diverse social and ethnic backgrounds who survived acute infection and attended a follow-up ambulatory clinic appointment. We identified that most patients experienced $\geq 1$ symptom for $\geq 120$ days after the onset of disease. This finding also applies to patients who had a mild or moderate form of COVID-19. These symptoms negatively affected the patients' quality of life; fatigue was the most common symptom, followed by dyspnea and cough.

The clinical picture we describe here, in a population with a mixed ethnic background consisting

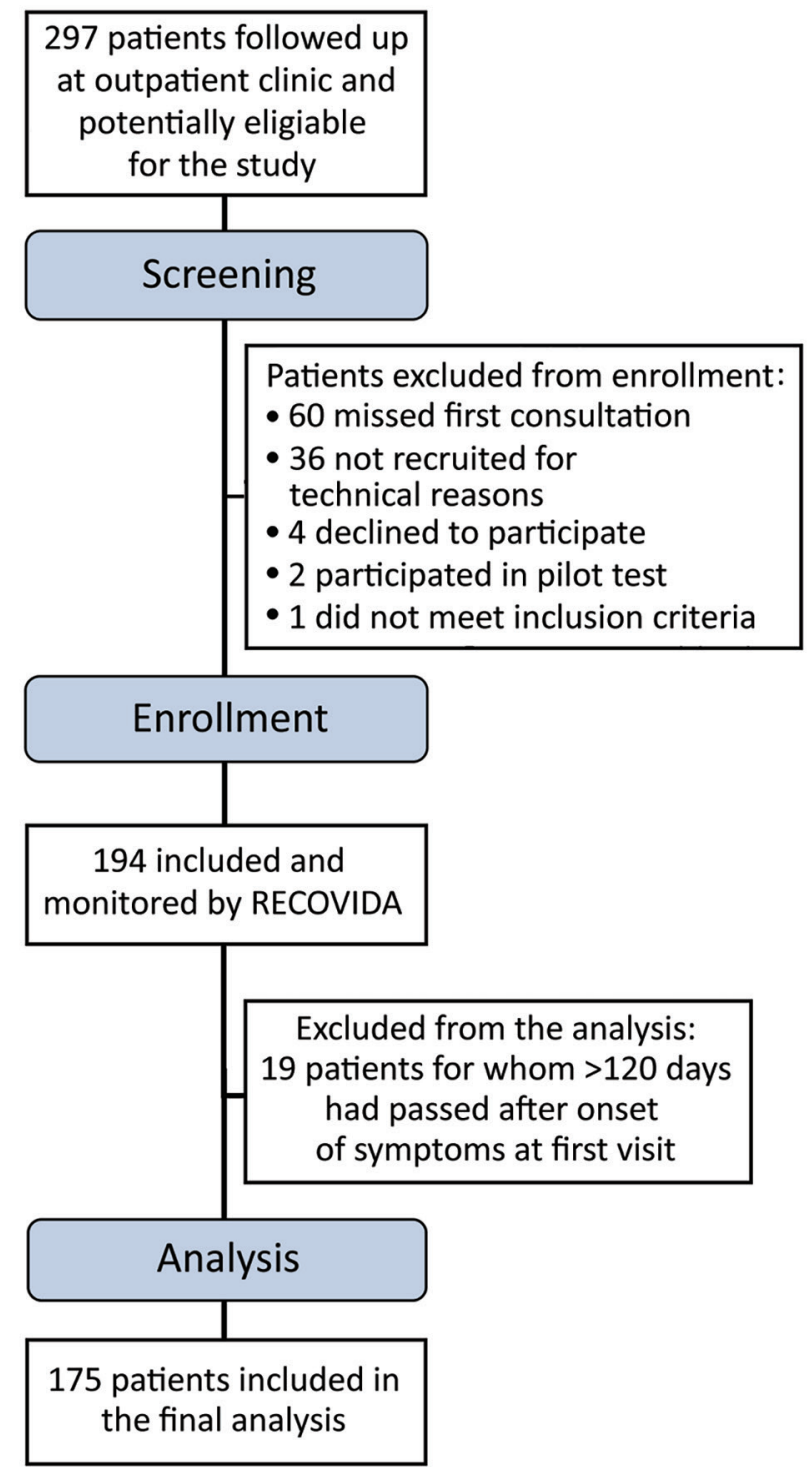

Figure. Flowchart of screening and inclusion of coronavirus disease survivors with long-term symptoms in prospective cohort study, Ribeirão Preto, Brazil. of Latin American, Caucasian, Afro-American, Asian, and Brazilian indigenous persons, is similar to those encountered in other parts of the world, mainly in Caucasian or Asian populations (1,1012). Some persistent symptoms found in our study, such as altered skin sensitivity and muscle weakness, primarily affected the patients whose illness was critical, and this finding could be more related to their stay in the intensive care unit than to the COVID-19 itself (13).

Several possible pathophysiological explanations for the persistence of symptoms after COVID-19 have been proposed. The most commonly elicited in the literature are direct viral toxicity, endothelial damage, dysregulated immune response, hyperinflammation, hypercoagulability, and poor adaptation of the angiotensin-converting enzyme 2 . So far, the actual mechanisms behind this scenario are not entirely understood and deserve further evaluation (1,10-13). Our sample identified that respiratory and heart rates were significantly higher in the patients whose illness was critical, possibly indicating impairment of autonomic function in these patients $(14,15)$.

We highlight the need to study the persistent symptoms of patients with COVID-19, given the implications for the healthcare system and social security, both of which are already profoundly affected by the pandemic itself. From this perspective, most persons with COVID-19 requiring medical consultation would not be expected to recover fully or resume working immediately after the end of the disease's acute phase. Instead, they will require a prolonged interdisciplinary healthcare approach focused on physical, mental, and social rehabilitation (1,10-15).

We did not perform genetic sequencing of the severe acute respiratory syndrome coronavirus 2 detected in our patients. Therefore, we cannot evaluate whether different virus variants might affect the occurrence of long-term symptoms among survivors differently.

One of the strengths of our study was our systematic follow-up on participants with prespecified instruments, which ensured high-quality and consistent data. A novelty of the study was that we were able to recruit patients who had mild or moderate COVID-19, which is less common in other studies.

A limitation of our study was the small sample size; the results therefore cannot be generalized to the wider population. Another limitation is the lack of a control group for comparison and selection bias. Most likely, many patients who did not attend a medical consultation after being discharged from the hospital experienced only mild or no prolonged 
symptoms at all. The same can be said for healthcare workers who were affected by COVID-19 but did not seek medical consultation. The actual prevalence of long-term symptoms among the reference population is unknown, and our data probably overestimate that prevalence.

In summary, it is likely that a substantial proportion of patients surviving COVID-19 will experience long-term symptoms requiring prolonged care, even after mild to moderate disease. These symptoms might negatively affect patients' quality of life and represent an additional burden for healthcare services and social security.

\section{Acknowledgments}

We thank the outpatient post-COVID-19 team (MINC) that gave us access to patients and offered the space for recruitment of study subjects and data collection; the surviving patients of COVID-19 who contributed to our work; and the research team of the Department of Social Medicine (Public Health) of the Ribeirão Preto Medical School, University of São Paulo (FMRP/USP). We would like to thank Editage (https:/ / www.editage.com) for their excellent input on the English language editing.

This work was partially supported by the Fundação de Apoio ao Ensino, Pesquisa e Assistência do Hospital das Clínicas da Faculdade de Medicina de Ribeirão Preto da Universidade de São Paulo (FAEPA), and National Council for Scientific and Technological Development (CNPq). L.P.B. was granted a scholarship from $\mathrm{CNPq}$ to work on Covid-19 research activities (process no. 309098/2020-3).

\section{About the Author}

Dr. Bonifácio is a physiotherapist and current postdoctoral student at the Department of Social Medicine, Ribeirão Preto Medical School, University of São Paulo, Brazil. Her primary areas of interest are primary care, public health and epidemiology, reproductive health, women's and men's health and gender and masculinities, orthopedics, and traumatology.

\section{References}

1. Nalbandian A, Sehgal K, Gupta A, Madhavan MV, McGroder C, Stevens JS, et al. Post-acute COVID-19 syndrome. Nat Med. 2021;27:601-15. https:/ / doi.org/10.1038/ s41591-021-01283-z

2. World Health Organization. Emergency use ICD codes for COVID-19 disease outbreak [cited 2021 May 3]. https://www.who.int/standards/classifications/ classification-of-diseases/emergency-use-icd-codes-forcovid-19-disease-outbreak
3. Havervall S, Rosell A, Phillipson M, Mangsbo SM, Nilsson P, Hober S, et al. Symptoms and functional impairment assessed 8 months after mild COVID-19 among health care workers. JAMA. 2021;325:2015-6. https:// doi.org/10.1001/jama.2021.5612

4. Instituto Brasileiro de Geografia e Estatística. Geographic and statistical overview of Ribeirão Preto [in Portuguese] [cited 2021 Mar 29]. https://cidades.ibge.gov.br/brasil/sp/ ribeirao-preto/panorama

5. World Health Organization. Global COVID-19 clinical platform case report form (CRF) for post COVID condition (Post COVID-19 CRF) [cited 2020 Oct 20]. https:/ / cdn.who. int/media/docs/default-source/3rd-edl-submissions/ who_crf_postcovid_feb9_2021.pdf

6. Harris PA, Taylor R, Thielke R, Payne J, Gonzalez N, Conde JG. Research electronic data capture (REDCap) - a metadata-driven methodology and workflow process for providing translational research informatics support. J Biomed Inform. 2009;42:377-81. https:/ / doi.org/10.1016/ j.jbi.2008.08.010

7. The WHOQOL Group. Development of the World Health Organization WHOQOL-BREF quality of life assessment. Psychol Med. 1998;28:551-8. https://doi.org/10.1017/ S0033291798006667

8. Fleck MP, Louzada S, Xavier M, Chachamovich E, Vieira G, Santos L, et al. Application of the Portuguese version of the abbreviated instrument of quality of life WHOQOL-bref [in Portuguese]. Rev Saude Publica. 2000;34:178-83. https:/ / doi.org/10.1590/S0034-89102000000200012

9. Pedroso B, Pilatti LA, Gutierrez GL, Picinin CT. Calculating WHOQOL-BREF scores and descriptive statistics through Microsoft Excel [in Portuguese]. Revista Brasileira de Qualidade de Vida. 2010;2:31-6. https:/ / doi.org/10.3895/ S2175-08582010000100004

10. Centers for Disease Control and Prevention. COVID-19: Long-term effects [cited 2021 May 3]. https:// www.cdc.gov/ coronavirus/2019-ncov/long-term-effects/index.html

11. Nature. Long COVID: let patients help define long-lasting COVID symptoms [editorial]. Nature. 2020;586:170. https:/ / doi.org/10.1038/d41586-020-02796-2

12. Nehme M, Braillard O, Chappuis F, Courvoisier DS, Guessous I; CoviCare Study Team. Prevalence of symptoms more than seven months after diagnosis of symptomatic COVID-19 in an outpatient setting. Ann Intern Med. 2021;174:1252-60. https://doi.org/10.7326/M21-0878

13. Seeßle J, Waterboer T, Hippchen T, Simon J, Kirchner M, Lim A, et al. Persistent symptoms in adult patients one year after COVID-19: a prospective cohort study. Clin Infect Dis. 2021 Jul 5 [Epub ahead of print].

14. Antwi-Amoabeng D, Beutler BD, Singh S, Taha M, Ghuman J, Hanfy A, et al. Association between electrocardiographic features and mortality in COVID-19 patients. Ann Noninvasive Electrocardiol. 2021;26:e12833. https:/ / doi.org/ 10.1111/anec.12833

15. Dixit NM, Churchill A, Nsair A, Hsu JJ. Post-acute COVID-19 Syndrome and the cardiovascular system: what is known? Am Heart J Plus. 2021;5:100025.

Address for correspondence: Fernando Bellissimo-Rodrigues or Lívia Pimenta Bonifácio, Social Medicine Department, Ribeirão Preto Medical School (USP), Campus Universitário, Monte Alegre 14048-900, Ribeirão Preto, São Paulo, Brazil; email: fbellissimo@ usp.br_or livia_pb@usp.br 